\title{
La Medicina y los Cuidados Paliativos
}

\author{
Francisco Javier Barón Duarte \\ Oncólogo del Complejo Hospitalario Universitario de Santiago \\ Coordinador del Plan Galego de Cuidados Paliativos
}

La puesta en marcha de programas de Cuidados paliativos en los planes de Salud es un indicador de la calidad de la atención e interés del sistema sanitario por el bienestar de sus ciudadanos.

En Galicia este interés puede evidenciarse mediante la puesta en Marcha del Plan Galego de Coidados Paliativos que es el marco para el desarrollo homogéneo y equitativo de los Cuidados Paliativos.

Este Plan presentado el 25 de abril del año 2006 supone no solamente un esfuerzo presupuestario para crear dispositivos de Cuidados Paliativos sino también un esfuerzo organizativo par mejorar la formación general en cuidados paliativos y la coordinación entre todos los medios y dispositivos del sistema sanitario.

Como se afirma en el documento "Bases para el desarrollo del Plan Nacional de Cuidados Paliativos "Todos los pacientes susceptibles de recibir cuidados paliativos están bajo la responsabilidad de la atención del conjunto de la red convencional de atención sanitaria; en situaciones de complejidad se requerirá la intervención de servicios específicos".

(Ministerio de Sanidad y Consumo. Plan Nacional de Cuidados Paliativos. Bases para su desarrollo. Madrid: Ministerio de Sanidad y Consumo, 2001.)

En la introducción del Plan Gallego de Cuidados Paliativos se afirma que "Implantar unidades o equipos de cuidados paliativos requiere combinar dos tipos de estrategias.

En primer lugar, la formación básica en cuidados paliativos para todos los profesionales sanitarios y, en segundo lugar, el desarrollo de programas específicos de cuidados paliativos con profesionales capacitados y con la posibilidad de intervención en todos los niveles asistenciales para mejorar notablemente la satisfacción de pacientes y familiares. Su implantación debe ser priorizada y planificada por la Administración sanitaria no solamente para mejorar la calidad de la atención para pacientes con patología progresiva y avanzada con pronóstico de vida limitado, sino también para mejorar la eficiencia global y el uso apropiado de lo recursos en el sistema público, en la busca de la equidad, garantizando que el acceso a estos cuidados sea semejante, sin distinción de territorio, recursos económicos o información."(Plan Galego de Coidados Paliativos pag.9. 2006)
Estudios desarrollados en países occidentales estiman que un $37,5 \%$ de los pacientes con necesidad de cuidados paliativos sufren situaciones intermedias o complejas que pueden requerir la intervención de un equipo específico de cuidados paliativos. El $60 \%$ restante tienen necesidades básicas de C. Paliativos atendidas por los dispositivos convencionales de la red sanitaria.

Así pues el Plan Galego de Coidados Paliativos se desarrolla simultáneamente en dos líneas estratégicas con la creación de dispositivos específicos de Cuidados Paliativos y con la mejor formación de profesionales y participación en acciones paliativas de todo el sistema sanitario.

En relación con la primera línea en el momento actual disponemos de 7 Unidades de Cuidados Paliativos en los 7 grandes Hospitales y de 3 Equipos de Soporte de Cuidados Paliativos en los Hospitales de la Costa, Monforte y Barco de Valdeorras. En los próximos meses se espera crear los Equipos de Soporte de Cuidados Paliativos en otros 4 Hospitales de modo que dispondremos de 14 dispositivos específicos de cuidados paliativos distribuidos equitativamente en nuestra a geografía. Las referencias internacionales sobre recursos de Cuidados Paliativos son 1 Equipo soporte accesible / sector (100.000-200.000) y 80-100 camas / millón: agudos, media, larga estancia.

Todavía no se han obtenido estas cifras y el reto de obtenerlas deberá guiar las actuaciones del Plan en el futuro.

En el ámbito de la segunda línea estratégica en el año 2007 más de 500 profesionales desarrollaron acciones formativas de Cuidados Paliativos, se constituyeron por primera vez en la Historia de la sanidad gallega 6 Comisiones Técnicas Sanitarias de Cuidados paliativos con profesionales de Atención Primaria y Hospitales y se nombraron 40 profesionales de Atención primaria como referentes de cuidados paliativos en sus Equipos de Atención primaria. Las Unidades de Hospitalización Domiciliaria trabajan de modo decisivo en el apoyo a la complejidad en la atención domiciliaria de los pacientes con enfermedades avanzadas, progresivas y complejas.

La visión moderna dos cuidados paliativos no se limita a atender a pacientes terminales sino que se extiende a todos los que presentan un pronóstico de vida mas o menos limitado por padecer enfermedades avanzadas, progresivas y complejas. 
Como define a Asociación Internacional de Hospices y Cuidados Paliativos "Los C.P. deben iniciarse cuando el paciente inicia una enfermedad sintomática, activa, progresiva e incurable. Nunca debe esperarse para a su aplicación a que los tratamientos específicos de la enfermedad estén agotados. Los cuidados paliativos son tratamientos activos y complementarios del tratamiento específico de la enfermedad de base. Deben integrarse "sin costura" con otros aspectos de los cuidados en la aproximación holística que atiende todos los aspectos do sufrimiento del paciente, aspecto que con frecuencia falta en la medicina orientada a la enfermedad". (IAHPC Manual of Palliative Care. 2nd Edition 2004)

Esta definición de cuidados paliativos se sustenta en el modelo biopsicosocial de atención sanitaria. El modelo biopsicosocial es a la vez una filosofía de los cuidados clínicos y una práctica clínica de los mismos.

Filosóficamente es una forma de entender como el sufrimiento y la enfermedad se afectan por múltiples niveles de organización, desde el social al molecular.

A nivel práctico es una forma de entender la experiencia subjetiva del paciente como una contribución esencial al adecuado diagnóstico, resultados de salud y cuidados de la persona.
(Francesc Borrell-Carrió, Anthony L. Suchman ,Ronald M. Epstein. TheBiopsychosocial Model 25 Years Later: Principles, Practice, and Scientific Inquiry. Ann Fam Med 2004; 2:576-582.)

Los principios de los Cuidados Paliativos tienen mucho en común con los de la Medicina Interna y las especialidades Médicas.

Así y ante los cambios producidos en el sistema asistencial, se plantea al médico el difícil problema de no dejar que se pierda el aspecto humano de la atención clínica. En Estados Unidos, la American Board of Internal Medicine, de acuerdo con el American College of Physicians-American Society of Internal Medicine y la Federación Europea de Medicina Interna, ha publicado su Charter on Medical Professionalism, que destaca los tres principios básicos en el compromiso del facultativo con la sociedad: 1) la prioridad del bienestar de la persona; 2) la autonomía del paciente, y 3) aspectos de justicia social. Las virtudes humanísticas del médico deben incluir integridad, respeto y compasividad.

Los Cuidados Paliativos recuperan la filosofía original de la Medicina y promueven una organización sanitaria centrada en el paciente. Es responsabilidad de todos los profesionales sanitarios apoyar su desarrollo y participar en su aplicación. 